\title{
Cut Elimination for Monomial MALL Proof Nets*
}

\author{
Olivier Laurent \\ CNRS - Université Paris VII \\ Olivier.Laurentepps.jussieu.fr
}

\author{
Roberto Maieli \\ Università Roma III \\ maieli@uniroma3.it
}

\begin{abstract}
We present a syntax for MALL (multiplicative additive linear logic without units) proof nets which refines Girard's one. It is also based on the use of monomial weights for identifying additive components (slices). Our generalization gives the possibility of representing a kind of sharing of nodes which does not exist in Girard's nets. This sharing leads to the definition of a strong cut elimination procedure for MALL.

We give a correctness criterion which is proved to be stable by reduction and to give a sequentialization theorem with respect to the sequent calculus. Sequentialization is proved by showing that an expansion procedure allows us to unfold any of our proof nets into a Girard proof net.
\end{abstract}

\section{Introduction}

Since its inception, in 1987, linear logic (LL, [Gir87]) has changed the proof theoretical way of dealing with cut elimination. This task was traditionally carried out by means of sequent calculi with the consequence that the most part of these works were engrossed by tedious commutations of rules. This situation has changed with the accession of the new geometrical syntax for proofs, known as proof nets. Proof nets are parallel presentations of sequential proofs of linear logic: they quotient classes of equivalent proofs, modulo irrelevant permutations of derivation rules.

The standard key ingredients of a proof net syntax are:

- a graphical syntax (proof structures),

- a correctness criterion (defining proof nets among proof structures),

- an interpretation of the sequent calculus syntax,

- a cut elimination procedure.

* Partially supported by the CNR-CNRS Research Programme Interaction Complexity and the MIUR Research Programme Rete Italo-Francese di Ricerca in Logica e Geometria della Computazione.
The main properties under consideration for a good notion of proof nets are then the following:

- Soundness of the interpretation: the graphs associated with a sequent calculus proof, which are usually given as proof structures, are indeed proof nets.

- Stability of correctness under cut elimination.

- Sequentialization theorem: each proof net is the image of at least one proof of the sequent calculus.

- Functional interpretation: the interpretation of sequent-calculus proofs into proof nets is a function.

- Canonical representation of proofs of the sequent calculus: proofs equal up to (reasonable) commutations of rules are identified (this leads to the representation of the corresponding free categories with proof nets).

- Completeness of cut elimination: for any cut node in a proof net a cut elimination step can be applied.

- Locality of cut elimination: a cut elimination step only affects the nodes connected to the cut node it is reducing.

- Strong normalization and confluence of the cut elimination procedure.

- Linear complexity of the correctness checking with respect to the number of nodes.

One usually requires at least the first two properties to hold, otherwise it is really difficult to consider the proposed proof-net syntax as a real alternative to the sequent calculus. Concerning proof nets, MLL (the Multiplicative fragment of LL) is the perfect setting: all these conditions are satisfied!

A lot of work has been done to extend these properties to MALL (the Multiplicative Additive fragment of LL). The syntax proposed by J.-Y. Girard in [Gir96] is a very important progress: a new (additive boxes free) syntax for proof nets of MALL where each node of a proof structure is weighted by a nonzero monomial (conjunctions or products) of a Boolean algebra generated by the (different) eigenweights (variables) associated to the \& nodes.

Unfortunately Girard's proposal is not as good as for MLL:

"Contrarily to the multiplicative case, the extant 


\section{solution is not perfect (although it has the virtue to exist)." \\ [Gir96, page 24]}

In particular the "canonical representation" property and the "functional interpretation" property are lost. The monomiality constraint imposed on the additive weights prevents from the natural representation of some sequent calculus proofs. There exist proofs with two possible associated proof nets with no way to discriminate them.

This problem has been solved by D. Hughes and R. van Glabbeek in a perfectly satisfactory way [HvG03]. By showing how to deal with non-monomial weights in the correctness criterion, they proposed a notion of additive proof net based on the formula trees of the conclusions of the proof decorated with families of atomic axiom links (linkings). This corresponds to a maximal gluing of slices from conclusions up to the axioms. The key point with respect to Girard's work is that this gluing was out of the scope of monomial weights. Hughes and van Glabbeek's nets satisfy all the properties mentioned before (except maybe the complexity one which is still an open question - as far as we know - and the locality of cut elimination that we will address below).

Let us mention two reasons why their nets can be bigger than Girard's nets. First, when interpreting a tensor rule of the sequent calculus (and the same for a cut rule), if the left premise induces $n$ slices (thus $n$ linkings) and the right one induces $m$ slices then the resulting proof net has $n \cdot m$ slices. Second, axioms are required to be atomic. As a consequence, a proof containing an axiom rule introducing a compound formula must be expanded before being translated into a proof net (this is perfectly natural from a categorical perspective, for example, since the categorical interpretation is invariant under axiom expansion). In particular, when one wants to work with non-expanded axioms (this can have consequences on complexity questions for example) or in extended settings where two disjoint formula occurrences in a sequent calculus proof can be related with the same sub-formula of the conclusions (as opposed to what happens in MALL), one could prefer to use Girard style proof nets.

We now look at cut elimination. Cut elimination with additive connectives naturally involves erasure and duplication of whole sub-proofs, that is non-local operations:

"Multiplicative proof nets have a local cutelimination [...] The additive case involves a real global move, which consists in setting an eigenweight to 0 or 1 , and erase everything with weight 0. This is rather brutal, and completely foreign to the parallel asynchronous spirit of proof-nets. [...] This is perhaps the most promising open question in this paper." [Gir96, page 26]

In [HvG03], everything happens at the level of axioms (because of the constraint on expansion of axioms): axioms can be duplicated (this is done in a local manner) or whole "inconsistent" linkings can be erased. This erasure in not a local operation but, in contrast, a completely global one: the whole linking is erased if it is inconsistent (moreover consistency is checked locally). We consider this local/global procedure as a reasonable weakening of the locality property. What would really be "foreign to the spirit of proofnets" is something in between where a (possibly complex) sub-part of the net would have to be computed for applying a reduction step. In [Gir96], a lazy procedure is given which only permits to normalize proof nets without \& connectives in conclusions. This procedure applies global steps for erasure (see the quotation above) and avoids to deal with any kind of duplication by laziness.

Our goal is to turn the lazy cut elimination procedure defined by Girard into a complete one (this requires to deal with duplication). Our proposal is based on the monomiality condition and thus will not be satisfactory enough from the canonicity point of view. The procedure we propose works in a local/global approach. In particular duplication of sub-nets is done in a local manner (in the same spirit as duplication in interaction nets). If we keep the monomiality condition, the crucial point is to relax another one: two different \& nodes will now be allowed to share the same eigenweight (under some appropriate restriction). In order to preserve monomiality, we attach to proof nets a global set of equations in the algebra of weights which has to be updated during reduction (once again this is not a local operation on the graph part of the net but a global one). We prove the following key properties of cut elimination: stability of correctness, completeness of the procedure, strong normalization and confluence.

In Section 2 we present a refinement of the original Girard's syntax in such a way to obtain a class of proof nets that is stable under a general cut elimination procedure. Naively, the main novelty is given by the immediate and local treatment of those cut elimination steps involving additive contraction links as cut premises (Section 3). This procedure is, moreover, shown to preserve the correctness criterion (Section 3.1) and to be strongly normalizing (Section 3.2).

A proof net is now defined as a pair $\langle\pi, E\rangle$ where $\pi$ is essentially a proof structure à la Girard, with the exception that here the eigenweights associated to the \& nodes are not supposed to be different; moreover, all weights must be monomial modulo $E$, that is, a set of null equations which mainly guarantees that $\pi$ is still a proof structure after the reduction.

Finally, we prove the sequentialization (Section 4) of our proof nets by means of an expansion procedure (Section 4.1) which allows us to unfold any proof net into a (unique) proof net à la Girard for which a sequentialization 
was already given: the expansion reestablishes the particular case of a proof structure where all \& nodes have different eigenweights.

MALL is the multiplicative and additive fragment of linear logic. Formulas $A, B, \ldots$ are built from literals (propositional variables $P, Q, \ldots$ and their negations $P^{\perp}, Q^{\perp}, \ldots$ ) by the binary connectives $\otimes$ (tensor), 8 (par), \& (with) and $\oplus$ (plus). Negation $(.)^{\perp}$ extends to arbitrary formulas by the de Morgan laws: $(A \otimes B)^{\perp}=\left(A^{\perp} 8 B^{\perp}\right)$, $(A \& B)^{\perp}=\left(A^{\perp} \otimes B^{\perp}\right),(A \& B)^{\perp}=\left(A^{\perp} \oplus B^{\perp}\right)$, and $(A \oplus B)^{\perp}=\left(A^{\perp} \& B^{\perp}\right)$. A MALL sequent $\Gamma$ is a set of formula occurrences $A_{1}, \ldots, A_{n}$. We omit turnstiles $(\vdash)$ since all sequents are right-sided. Sequents are proved using the following rules:

$$
\begin{aligned}
& \overline{A, A^{\perp}} \text { ax } \frac{\Gamma, A \quad \Delta, A^{\perp}}{\Gamma, \Delta} \mathrm{cut} \\
& \frac{\Gamma, A \quad \Delta, B}{\Gamma, \Delta, A \otimes B} \otimes \quad \frac{\Gamma, A, B}{\Gamma, A \ngtr B} 8 \\
& \frac{\Gamma, A \quad \Gamma, B}{\Gamma, A \& B} \& \quad \frac{\Gamma, A}{\Gamma, A \oplus B} \oplus_{1} \quad \frac{\Gamma, B}{\Gamma, A \oplus B} \oplus_{2}
\end{aligned}
$$

\section{MALL Proof structures}

The following notion of proof structure is essentially a refinement of that one given in [Gir96] (see Appendix A) but, for technical reasons, we adopt the syntax of [Lau99], with explicit $n$-ary additive contraction nodes $(C)$. Naively, a MALL proof structure is an oriented graph (pre-proof structure) with associated weights.

DEFINITION 1 A MALL pre-proof structure (PPS) $\pi$ is a non-empty directed graph such that each edge is labelled by a MALL formula and built on the set of nodes following the typing constraints of Figure 1:

1. fixed a node, an entering edge is called premise while its emergent edges are called conclusions;

2. each edge must be conclusion of exactly one node and premise of at most one node;

3. we assume $A=A_{1}=\ldots=A_{n \geq 1}$ in the additive contraction $(C)$ node;

4. two contraction nodes cannot have a common edge.

Pending edges are called conclusions of $\pi$. We call link the graph made by a node together with its premise(s) and its conclusion(s) (if any).

Definition 2 Assume a set $\mathcal{B}$ of Boolean variables denoted by $p, q, \ldots$, then a monomial weight (simply, a weight) $w, v, \ldots$ over $\mathcal{B}$ is a product (conjunction) of variables or negation of variables of $\mathcal{B}$. We replace p.p by $p$. Often, in a product of weights, $v$ and $w$, we omit "." and we

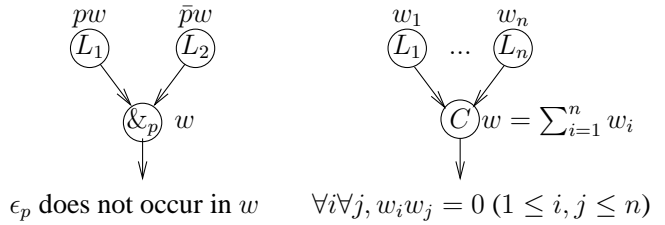

Figure 2. Weights for $\&$ and $C$ links

write "vw" instead of "v.w". As usual in a Boolean algebra, we define the standard order relation " $\leq$ " between two weights $v$ and $w$ as follows: $v \leq w$ if there exists a weight $v^{\prime}$ s.t. $v=v^{\prime}$.w. Moreover, we assume the following notation: 1 , for the empty product; 0 , for a product where both $p$ and $\bar{p}$ appear; $\epsilon_{p}$, for a variable p or its negation $\bar{p}$; then we use the special product notation $\epsilon_{p} @ v$, equivalent to $\epsilon_{p} . v$, when we want to focus on the prefix weight $\epsilon_{p}$ within the weight $\epsilon_{p} . v$ ( $v$ is said suffix weight). We say that a weight $w$ depends on a variable $p$ when $\epsilon_{p}$ appears in $w$; we say that two weights, $v$ and $w$, are disjoint when $v . w=0$.

DEFINITION 3 A MALL proof structure (PS), is a pair $\langle\pi, E\rangle$ such that:

1. E is a set of null equations of the shape $\epsilon_{p} @ w=0$ where $\epsilon_{p}$ is a prefix weight over $\mathcal{B}$ and $w$ is a weight over $\mathcal{B}$ not containing any occurrence of $p$ or $\bar{p}$;

2. $\pi$ is a MALL pre-proof structure in which we associate a Boolean variable $p$ over $\mathcal{B}$, called eigenweight, to each \& node of $\pi$ and we use the notation $\&_{p}$; eigenweights are not supposed to be different. Then, to each node we associate a nonzero, up to $E$, weight $w$ of the Boolean algebra generated over $\mathcal{B}=\left\{p \mid \&_{p} \in\right.$ $\pi \vee \epsilon_{p}$ is a prefix in $\left.E\right\}$ with the constraint that two nodes have the same weight if they have a common edge, except when the edge is the premise of a \& or $C$ node; in these cases we do as follows (see Figure 2):

(a) if $w$ is the weight of a \& node and $p$ is its eigenweight then $w$ does not depend on $p$ and its premise nodes must have weights pw and $\bar{p} w$;

(b) if $w$ is the weight of a $C$ node and $w_{1}, \ldots, w_{n}$ are the weights of its premise nodes, then we must have $w=\sum_{i=1}^{n} w_{i}$, modulo $E$, with $w_{i} . w_{j}=0$ and $w_{i} \leq w, \forall i, j$ s.t. $1 \leq i<j \leq n$;

3. each conclusion node has weight 1 ;

4. if $w$ is a weight of a node appearing in $\pi$ or a weight occurring in an equation $w=0$ of $E$ and $w$ depends on $p$, then

$$
w \leq\left(\sum_{i=1}^{n} v_{i}\right) \quad \bmod E
$$



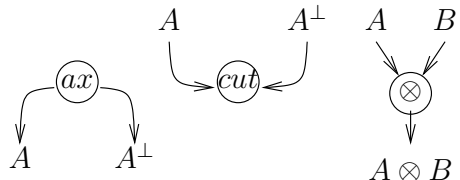

$A \otimes B$
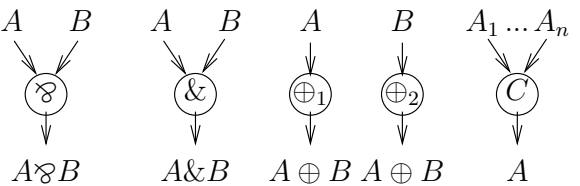

Figure 1. MALL links

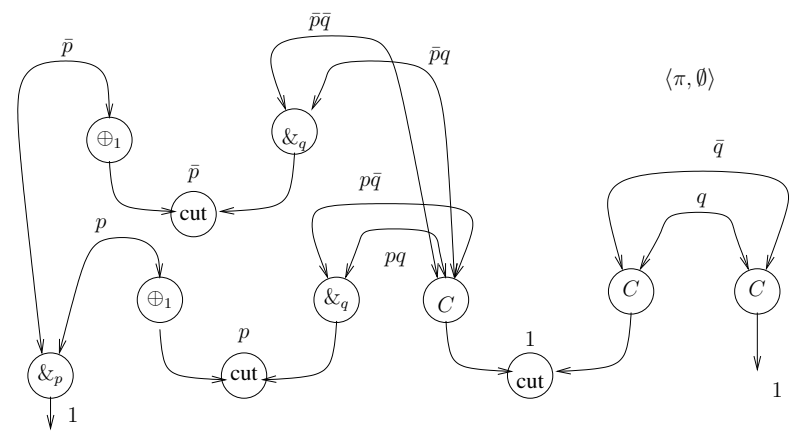

Figure 3. Example of proof structure

where $v_{i}$, with $1 \leq i \leq n$, is either the weight of $a \&_{p}$ node or the suffix weight of an equation $\epsilon_{p} @ v_{i}=0$ of E; moreover,

(a) $\sum_{i=1}^{n} v_{i}$ must be a monomial weight, up to $E$;

(b) all weights $v_{1}, \ldots, v_{n}$ must be pairwise disjoint.

A node $L$ with weight $w$ depends on $p$ if $w$ depends on $p$.

As simplification of the graph notation we may sometimes use, in the following, left-right arrow edges $(\leftrightarrow)$ to denote axiom links. We also omit sometimes the edge orientation when it is clear from the context.

EXAMPLE 1 In Figure 3 we give an instance of proof structure: the pair $\langle\pi, \emptyset\rangle$.

We are interested in those proof structures that correspond to proofs of the sequent calculus; these proof structures will be called correct proof structures or proof nets.

DEFINITION $4 A$ valuation $\varphi$ for a $P S\langle\pi, E\rangle$ is a function that maps each eigenweight of $\pi$ (resp., each prefix weight of $E)$ into $\{0,1\}$; this mapping must be $E$-consistent, that is, if an equation $\epsilon_{p} @ v=0$ occurs in $E$, then $\varphi\left(\epsilon_{p} v\right)=0$.

Fixed a valuation $\varphi$ for $\langle\pi, E\rangle$ then:

- a slice $\varphi(\pi)$ is the graph obtained from $\pi$ by keeping only those nodes with weight 1 together with its emerging edges; a local slice $\varphi_{\text {loc }}(\pi)$ is a slice $\varphi(\pi)$ where we replaced each occurrence of $\epsilon_{p}$ by $\epsilon_{p} v$ where $v$ is (possibly) the weight of the (unique) $\&_{p}$ node appearing in the slice;
- $a$ multiplicative switching $S$ for $\pi$ (we write $S(\pi)$ ) is the non oriented graph built on the nodes and edges of $\varphi(\pi)$ with the modification that for each 8-node we take only one premise and we cut the remaining one (left or right 8 -switch);

- an additive switching (or simply a switching) is a multiplicative switching in which for each \& node we cut the (unique) premise in $\varphi(\pi)$ and we add an oriented edge, called jump, from the \& node to a $L$ node whose weight depends on the eigenweight of this \& node. A switching $S$ for $\pi$ is called local when it is considered w.r.t. a local slice $\varphi_{l o c}(\pi)$.

DEFINITION 5 A proof structure $\langle\pi, E\rangle$ is correct, so it is a proof net (PN), if any local switching induced by a valuation $\varphi$ for $\langle\pi, E\rangle$ is acyclic and connected (ACC).

\section{Cut elimination}

DEFINITION 6 Let $L$ be a cut node in a proof structure $\langle\pi, E\rangle$ whose premises $A$ and $A^{\perp}$ are the respective conclusions of nodes $L^{\prime}$ and $L^{\prime \prime}$ (with $L^{\prime} \neq L^{\prime \prime}$ ); then we define the result $\left\langle\pi^{\prime}, E^{\prime}\right\rangle$ of reducing this cut in $\langle\pi, E\rangle$ (we write $\left.\langle\pi, E\rangle \rightsquigarrow_{L}\left\langle\pi^{\prime}, E^{\prime}\right\rangle\right)$ according to the following reduction steps.

(ax)-step : If $L^{\prime}$ (resp., $\left.L^{\prime \prime}\right)$ is an axiom node of $\pi$, then $\langle\pi, E\rangle \rightsquigarrow\left\langle\pi^{\prime}, E\right\rangle$, where $\pi^{\prime}$ is obtained by removing in $\pi$ both formulas $A$ and $A^{\perp}$ (as well as $L$ ) and giving as new conclusion to $L^{\prime \prime}$ (resp., $\left.L^{\prime}\right)$, the other conclusion of $L^{\prime}$ (resp., $\left.L^{\prime \prime}\right)$, like in Figure 4. If $L^{\prime \prime}$, in $\pi^{\prime}$, is a

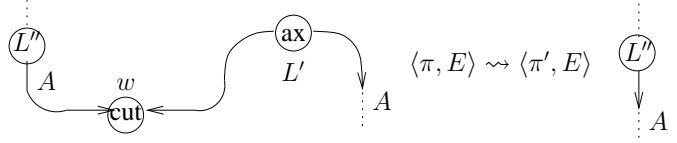

Figure 4. $a x$-cut reduction step

contraction node whose conclusion A is premise of an other contraction node, then, by associativity, we collapse these two contraction nodes into a unique one. 
( $\otimes / 8)$-step : If $L^{\prime}$ is $a \otimes$ node with premises $B$ and $C$ and $L^{\prime \prime}$ is a 8 node with premises $B^{\perp}$ and $C^{\perp}$, then $\langle\pi, E\rangle \rightsquigarrow\left\langle\pi^{\prime}, E\right\rangle$, where $\pi^{\prime}$ is obtained by removing in $\pi$ the formulas $A$ and $A^{\perp}$ as well as the cut node $L$ with $L^{\prime}$ and $L^{\prime \prime}$ and by adding two new cut nodes with premises, respectively, $B, B^{\perp}$ and $C, C^{\perp}$, like in Figure 5.

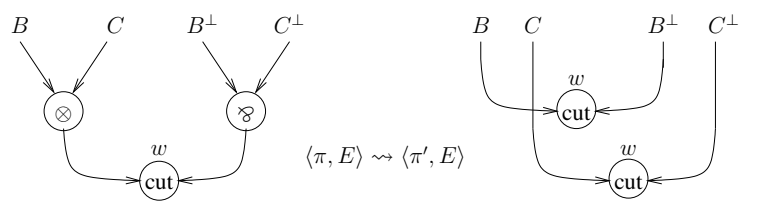

Figure 5. $(\otimes / 8)$-cut reduction step

$\left(\oplus_{i} / \&\right)$-step : If $L^{\prime}$ is a $\&_{p}$ node with weight $w$ and $B$ and $C$ as premises whose weights are, respectively, pw and $\bar{p} w$, and $L^{\prime \prime}$ is a $\oplus_{1}$ node with premise $B^{\perp}$ in $\pi$, then $\langle\pi, E\rangle \rightsquigarrow\left\langle\pi^{\prime}, E^{\prime}\right\rangle$, like in Figure 6, where:

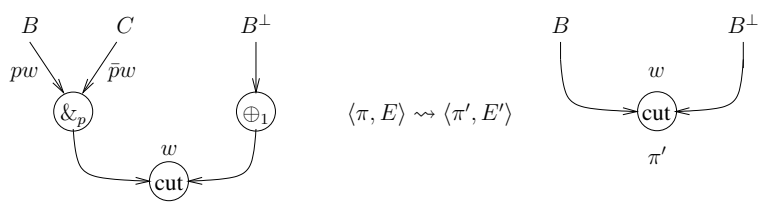

Figure 6. $\left(\& / \oplus_{1}\right)$-cut reduction step

1. $\pi^{\prime}$ is obtained by

(a) removing from $\pi$ any node whose weight $v$ is s.t. $v \leq \bar{p} w$ together with $L, L^{\prime}$ and $L^{\prime \prime}$,

(b) replacing each occurrence of the weight pw with $w$,

(c) adding a cut between $B$ and $B^{\perp}$;

2. $E^{\prime}$ is obtained by adding to $E$ all the equations $\epsilon_{q} @ v=0$ s.t. $\epsilon_{q} v \leq \bar{p} w$, for every $q$ that is eigenweight of a \& node of $\pi$ whose weight is $v$ (in particular, $\bar{p} @ w=0 \in E^{\prime}$ ).

The $\left(\oplus_{2} / \&\right)$-cut reduction step is analogous.

$(\otimes / C)$-step : If $L^{\prime}$ is a $C$ node and $L^{\prime \prime}$ is a $\otimes$ node, then $\langle\pi, E\rangle \rightsquigarrow\left\langle\pi^{\prime}, E\right\rangle$ where $\pi^{\prime}$ is obtained by replacing in $\pi$ the (sub-)graph of Figure 7 with that one of Figure 8.

(8/C)-step : Analogous to the previous one (where we replace each occurrence of $\otimes$ with an occurrence of 8$)$.

$\left(\oplus_{i} / C\right)$-step : If $L^{\prime}$ is a $C$ node and $L^{\prime \prime}$ is a $\oplus_{i}$ node, then $\langle\pi, E\rangle \rightsquigarrow\left\langle\pi^{\prime}, E\right\rangle$, where $\pi^{\prime}$ is obtained by replacing

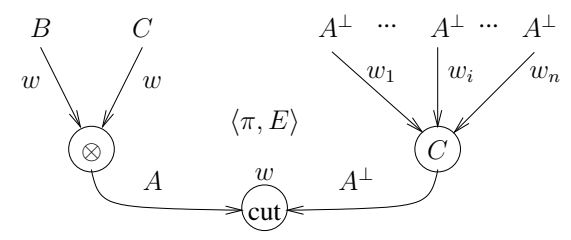

Figure 7.

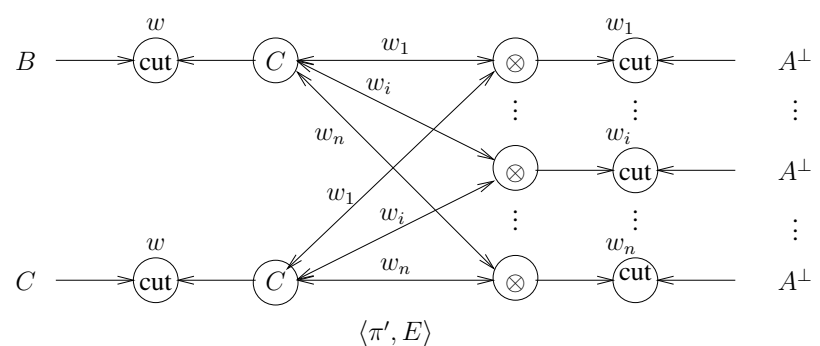

Figure 8.

in $\pi$ the (sub-)graph of Figure 9 with that one of Figure 10.

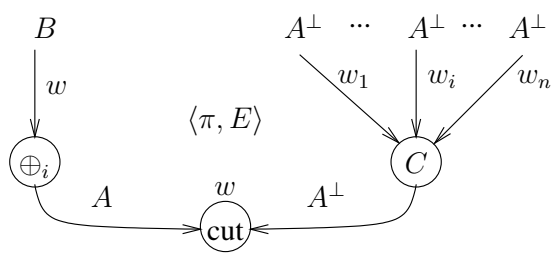

Figure 9.

$(\& / C)$-step : If $L^{\prime}$ is a $\&_{p}$ node and $L^{\prime \prime}$ is a $C$ node, then $\langle\pi, E\rangle \rightsquigarrow\left\langle\pi^{\prime}, E\right\rangle$ where $\pi^{\prime}$ is obtained by replacing in $\pi$ the sub-graph of Figure 11 with that one of Figure 12 (we keep only those axioms with nonzero weight).

$(C / C)$-step : If both $L^{\prime}$ and $L^{\prime \prime}$ are $C$ nodes, then $\langle\pi, E\rangle \rightsquigarrow\left\langle\pi^{\prime}, E\right\rangle$ where $\pi^{\prime}$ is obtained by replacing in $\pi$ the sub-graph of Figure 13 with that one of Figure 14 (we keep only those axioms with nonzero weight).

EXAMPLE 2 Reducing the two instances of a $\&_{q} / \oplus_{1}$ cut in the proof structure $\langle\pi, \emptyset\rangle$ of Figure 3 leads, respectively, to the proof structures $\left\langle\pi^{\prime},\{\bar{q} @ \bar{p}=0\}\right\rangle$ and $\left\langle\pi^{\prime \prime},\{\bar{q} @ \bar{p}=\right.$ $0, \bar{q} @ p=0\}\rangle$ of Figure 15: observe that the set of equations $\{\bar{q} @ \bar{p}=0, \bar{q} @ p=0\}$, in the last one, forces $\bar{q}=0$. 


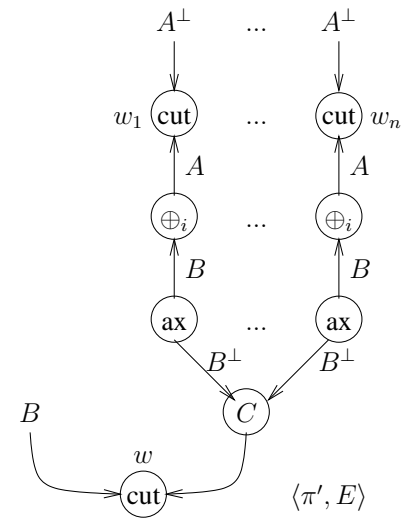

Figure 10.

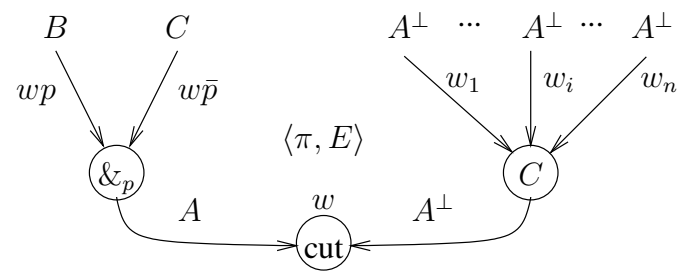

Figure 11.

\subsection{Stability}

In this section we show that correctness of proof structures is stable (preserved) under the cut reduction steps.

LEMMA 1 (\&-MUTILATION) Let $\langle\pi, E\rangle$ be a proofnet and let $L$ be a cut with weight $w$ between a $\&_{p}$ node and an $\oplus_{i}$ node $(i=1,2)$ and assume $\langle\pi, E\rangle \rightsquigarrow_{L}\left\langle\pi^{\prime}, E^{\prime}\right\rangle$; then there does not exist any mutilated (i.e. unary) \& node in $\pi^{\prime}$.

Proof - Assume $L$ is a cut with weight $w$ having $\&_{p}$ and $\oplus_{1}(i=1)$ as premises and assume $\langle\pi, E\rangle \rightsquigarrow_{L}\left\langle\pi^{\prime}, E^{\prime}\right\rangle$, with $E^{\prime}$ like in Definition 6. Assume, by absurdum, there exists a node $\&_{q}$, in $\pi$, whose weight is $v$ and s.t. it becomes unary after the $L$ reduction; this means that, by construction of $\pi^{\prime}, q v \leq \bar{p} w$. Now observe that it cannot be $\bar{p}=q$ otherwise, by definition of proof structure, it should be $v \cdot w=0$, contradicting $v \leq w$; Then, $\bar{p} w$ must occur in $v$, by the order relation " $\leq$ " on weights; this implies $v=0$, contradicting the assumption that the unary node $\&_{q}$ occurs (with nonzero weight) in $\pi^{\prime}$.

THEOREM 1 (STABILITY OF CUT REDUCTION) If a proof net $\langle\pi, E\rangle$ reduces in one step to $\left\langle\pi^{\prime}, E^{\prime}\right\rangle$, then $\left\langle\pi^{\prime}, E^{\prime}\right\rangle$ is still a proof net.

Proof - We reason by cases.

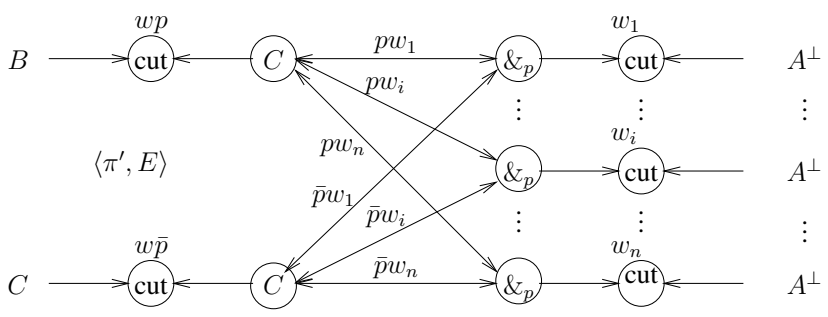

Figure 12.

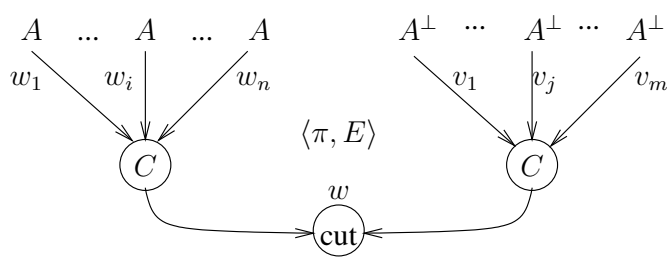

Figure 13.

1. If $\langle\pi, E\rangle$ reduces to $\left\langle\pi^{\prime}, E^{\prime}\right\rangle$ by an instance of a $a x,(8 / \otimes),(\otimes / C),(8 / C),\left(C / \oplus_{i}\right)$ or $(C / C)$-step of cut reduction, then it is trivial to verify that $\left\langle\pi^{\prime}, E^{\prime}\right\rangle$, having $E^{\prime}=E$, is still a proof structure; moreover, each local switching $S\left(\pi^{\prime}\right)$ can be seen as a sub-graph (possibly, collapsing the resulting unary nodes) of a fixed switching for $\pi$.

2. If $\langle\pi, E\rangle$ reduces to $\left\langle\pi^{\prime}, E^{\prime}\right\rangle$ by an instance of $\left(\oplus_{1} / \&_{p}\right)$ cut reduction step (see Figure 6) then it is easy to verify that, up to $E^{\prime},\left\langle\pi^{\prime}, E^{\prime}\right\rangle$ is still a proof structure (recall that $w=p w$, modulo $E^{\prime}$ ); in particular, it is easy to verify that the $\left(\oplus_{1} / \&_{p}\right)$ reduction step preserves the technical condition (4) of Definition 3, moreover Lemma 1 ensures that we do not mutilate any \& node except that one that is premise of the cut involved in the reduction. Finally, $\left\langle\pi^{\prime}, E^{\prime}\right\rangle$ is still correct since each local switching $S\left(\pi^{\prime}\right)$ can be seen as a sub-graph of a fixed switching for $\pi$.

3. Assume $\langle\pi, E\rangle$, like in Figure 11, reduces to $\left\langle\pi^{\prime}, E^{\prime}\right\rangle$ by a $\left(\&_{p} / C\right)$ cut reduction step, like in Figure 12; first, it is easy to verify that $\left\langle\pi^{\prime}, E^{\prime}\right\rangle$ is still a proof structure; then, in order to show that each local switching $S\left(\pi^{\prime}\right)$ is ACC we reason by absurdum.

Acyclic - Assume there exists a local switching $S\left(\pi^{\prime}\right)$ containing a cycle $\sigma$; then we need to consider two sub-cases, depending on the fact that we replace in any local switching for $\pi^{\prime}$ the occurrences of $\epsilon_{p}$ by the weight $\epsilon_{p} w$, where $w$ is the weight of the $\&_{p}$ node involved in the reduction.

- First case: $\sigma$ does not contain any jump $J$ from a 


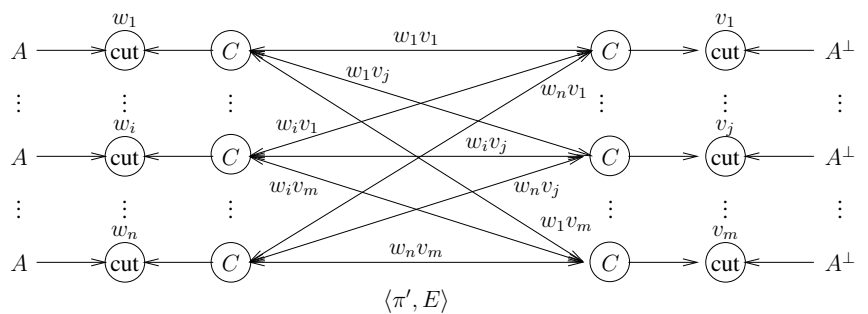

Figure 14.

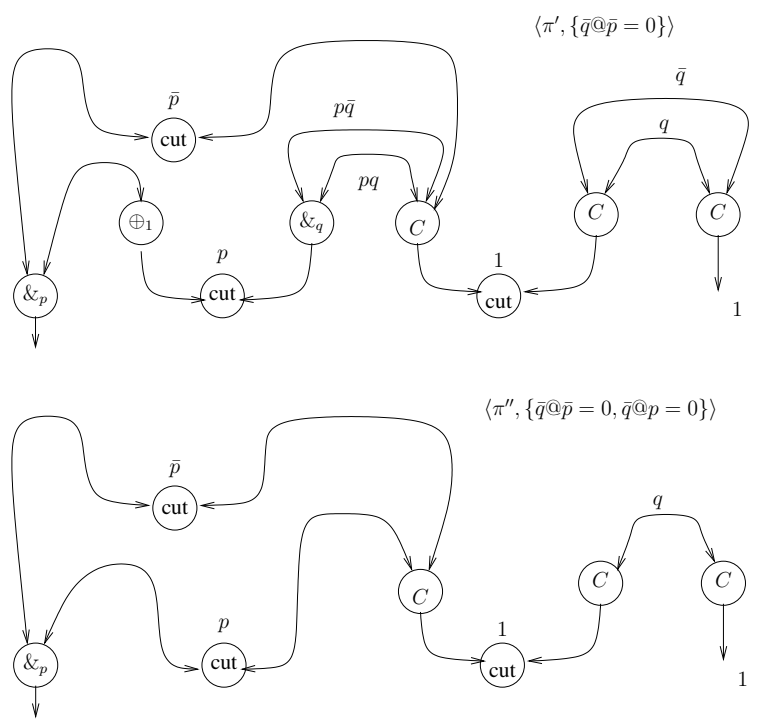

Figure 15. Examples of cut reduction

node $\&_{q}$ to a node $L$ such that its (local) weight $v$ depends on $q$ in the local switching $S\left(\pi^{\prime}\right)$ but it does not depend on $q$ in any local switching $S$ for $\pi$. This means that the local switching $S\left(\pi^{\prime}\right)$ can be seen as a subgraph of some local switching $S$ for $\pi$; so we get a contradiction, since by assumption $\langle\pi, E\rangle$ is correct.

- Second case: $\sigma$ contains a jump $J$ from a $\&_{q}$ node to a node $L$ such that its (local) weight $v$ depends on $q$ in $S\left(\pi^{\prime}\right)$ but it does not depend on $q$ in any switching $S$ for $\pi$. This means that $v$ has been obtained by replacing in the local slice each occurrence of $p$ (resp., $\bar{p}$ ) by an instance of $p w_{i}$ (resp., $\bar{p} w_{i}$ ), where $w_{i}$ is the weight of the (unique) premise of the the $C$ node involved in the cut reduction; clearly, $w_{i}$ depends on $q$, since it cannot be $p=q$. Now, independently of the fact that $\&_{p}$ node occurs or not in the cycle $\sigma$ of $S\left(\pi^{\prime}\right)$, we can suppose a new switching $S$ for $\pi$ that is identical to $S$ for $\pi^{\prime}$ except for the fact that one of the sub-graphs of those (A, B or C) depicted on the top of Figure 16 is replaced by the corresponding one on the bottom of the same Figure 16 (we use the notation $v\left[v^{\prime \prime} / v^{\prime}\right]$ to denote the substitution in $v$ of $v^{\prime}$ with $v^{\prime \prime}$ ). In $S(\pi)$ the jump $J$ goes from the $\&_{q}$ node to the (unique) premise (with weight $w_{i}$ ) of $C$; then, we get a local switching ( $S$ for $\pi$ ) that contains clearly a cycle, contradicting the assumption $\langle\pi, E\rangle$ is correct.

Connected - Assume that there exists a local switching $S\left(\pi^{\prime}\right)$ that is not connected; clearly this disconnection must be consequence of the fact that we jump from a node $\&_{q}$ to a node $L$ such that its local weight $v$ depends on $q$ in the local switching $S\left(\pi^{\prime}\right)$ but it does not depend on $q$ in any local switching $S$ for $\pi$ (otherwise $S\left(\pi^{\prime}\right)$ would be a sub-graph of some local switching $S$ for $\pi$ contradicting the correctness of $\langle\pi, E\rangle$ ). So, assume a local switching $S\left(\pi^{\prime}\right)$ with at least two separated components, $\alpha$ and $\beta$, such that $\alpha$ (or $\beta$ ) contains a jump $J$ from the $\&_{q}$ to such a node $L$. Now, let us suppose an other switching $S^{\prime}$ for $\pi^{\prime}$ that is identical to $S\left(\pi^{\prime}\right)$ except for the immediate jump from $\&_{q}$ to its (unique) premise. Clearly this switching must be connected, otherwise we could easily find a switching for $\pi$ containing $S^{\prime}\left(\pi^{\prime}\right)$, contradicting the assumption $\langle\pi, E\rangle$ is correct. Now, in $S^{\prime}\left(\pi^{\prime}\right)$, there must exist a path connecting $L$ to $\beta$; this path cannot contains $\&_{q}$ for the following reasons:

(a) it cannot start from $L$ and enter $\&_{q}$ from its conclusion, otherwise there would already exist in $S\left(\pi^{\prime}\right)$ a cycle containing $\&_{q}$;

(b) it cannot start from $L$ and enter $\&_{q}$ from its unique premise in $S^{\prime}\left(\pi^{\prime}\right)$, otherwise $\&_{q}$ and $\beta$ would already be connected in $S\left(\pi^{\prime}\right)$.

Therefore, in $S\left(\pi^{\prime}\right)$, a jump from a $\&_{q}$ to $L$ (the only thing that differs $S$ from $S^{\prime}$ ) cannot break the path connecting $L$ and $\beta$, contradicting our assumption.

REMARK 1 We could simplify the condition 2 of the $\oplus_{i} / \&$ reduction step of Definition 6 as follows:

$$
E^{\prime}=E \cup\{\bar{p} @ w=0\}
$$

where we actually enlarge $E$ only with the equation $\bar{p} @ w=$ 0 (i.e., the unique equation having as prefix the eigenweight of the \& node involved in the cut reduction). It is easy to show that the Definition 3, is stable under this new $\oplus_{i} / \&$ cut reduction step (where we replaced the condition 2 with the new one expressed by the equation 2 above). Indeed, this new cut reduction step preserves the correction itself.

However, for technical reasons connected to the sequentialization (especially, the expansion technique of Section 4) we prefer to retain the original definition. 


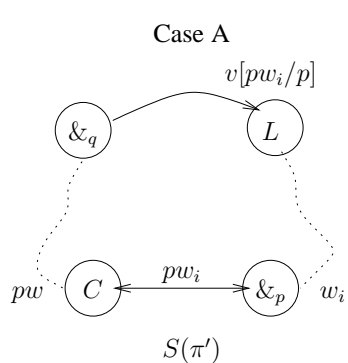

I

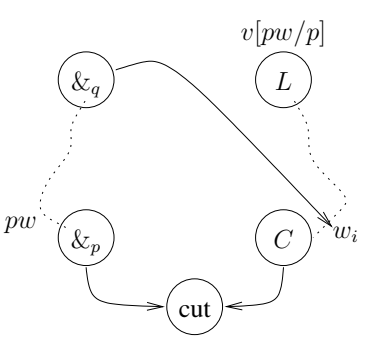

$S(\pi)$

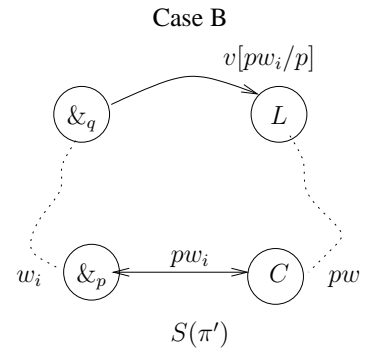

I

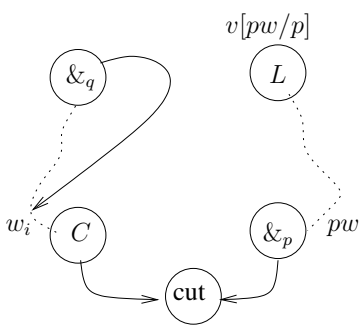

$S(\pi)$

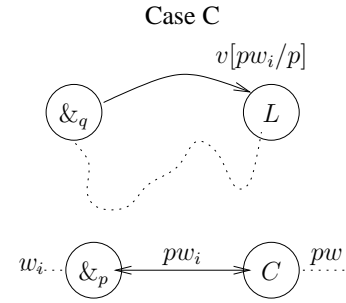

$S\left(\pi^{\prime}\right)$

I

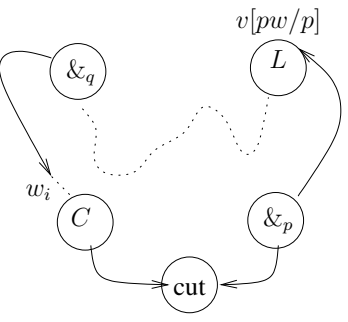

$S(\pi)$

Figure 16.

\subsection{Strong cut elimination}

THEOREM 2 (CONFLUENCE) Let $\langle\pi, E\rangle$ be a proof net with two cut nodes, $L_{1}$ and $L_{2}$, and let $\alpha$ be the cut reduction $\langle\pi, E\rangle \rightsquigarrow_{L_{1}}\left\langle\pi_{1}, E_{1}\right\rangle$ and $\beta$ be the cut reduction $\langle\pi, E\rangle \rightsquigarrow L_{2}\left\langle\pi_{2}, E_{2}\right\rangle$. Then, there exists a proof net $\left\langle\pi^{*}, E^{*}\right\rangle$ to which $\left\langle\pi_{i}, E_{i}\right\rangle$, for $1 \leq i \leq 2$, reduces in at most one step.

Proof - In general $\alpha$ and $\beta$ are independent, that is, they have local actions that do not interfere with each other except when at least one of them is a $\left(\& / \oplus_{i}\right)$ cut reduction step. So, assume $\alpha$ is a $\left(\&_{p} / \oplus_{1}\right)$ cut reduction step and assume $L_{1}$ with weight $w_{1}$ and $L_{2}$ with weight $w_{2}$, then there are two main cases:

First Case: $\beta$ is not a $\left(\& / \oplus_{i}\right)$ cut reduction step, then:

1. in case $w_{2} \leq \bar{p} w_{1}$ we have $\left\langle\pi_{2}, E_{2}\right\rangle \subseteq\left\langle\pi_{1}, E_{1}\right\rangle$;

2. otherwise, $\alpha$ and $\beta$ are independent and so we get $\left\langle\pi_{1}, E_{1}\right\rangle \rightsquigarrow L_{2}\left\langle\pi^{*}, E^{*}\right\rangle$ and $\left\langle\pi_{2}, E_{2}\right\rangle \rightsquigarrow L_{1}\left\langle\pi^{*}, E^{*}\right\rangle$;

Second Case: $\beta$ is a $\left(\&_{q} / \oplus_{1}\right)$ cut reduction step, then

1 . in case $p=q$ the two instances $\alpha$ and $\beta$ are independent and actually Lemma 1 ensures there is no interaction between them;

2. in case $p \neq q$, then: either $w_{2} \leq \bar{p} w_{1}$ (resp., $w_{1} \leq$ $\left.\bar{q} w_{2}\right)$ and so we are back to the case (1), or $\alpha$ and $\beta$ are independent (of course, by definition of proof structure, is not given the case of both $w_{2} \leq \bar{p} w_{1}$ and $\left.w_{1} \leq \bar{q} w_{2}\right)$.

THEOREM 3 (STRONG CUT ELIMINATION) We can always reduce a proof net $\langle\pi, E\rangle$ into a proof net $\left\langle\pi^{\prime}, E^{\prime}\right\rangle$ that is cut-free, by iterating the reduction steps of Definition 6; this reduction is strongly terminating.

Proof - If $s$ is a sequence of reductions from a proof net $\pi$, there exists a (at least as long) sequence $s^{\prime}$ of reductions from $\pi$ which does not contain any $\left(\& / \oplus_{i}\right)$-step erasing a cut link (we call such a sequence a cut-preserving sequence).

As a consequence, if we prove that we cannot find any infinite cut-preserving sequence of reductions in our proof nets, we obtain strong normalization.

For cut-preserving sequences, Theorem 2 can be strengthen: if $\langle\pi, E\rangle \rightsquigarrow L_{1}\left\langle\pi_{1}, E_{1}\right\rangle$ and $\langle\pi, E\rangle \rightsquigarrow L_{2}$ $\left\langle\pi_{2}, E_{2}\right\rangle$, there exists a proof net $\left\langle\pi^{*}, E^{*}\right\rangle$ to which $\left\langle\pi_{i}, E_{i}\right\rangle$, for $1 \leq i \leq 2$, reduces in one step. This means that weak normalization of cut-preserving reductions implies strong normalization of cut-preserving reductions.

We are going to prove weak normalization of unrestricted reduction in fact.

We show that, starting from a set $\mathcal{C}$ of cut links with disjoint weights and with the same logical complexity ${ }^{1}$ (which

\footnotetext{
${ }^{1}$ The complexity of a cut node is the logical complexity of its cutformula. The logical complexity of a formula is inductively defined as
} 
is called the logical complexity of $\mathcal{C}$ ), it is possible to reduce all of them by generating only cuts of strictly smaller logical complexity. This implies weak normalization by using the multiset ordering on the multiset of all the logical complexities of all the cut links of a proof net.

Given such a set $\mathcal{C}$ of logical complexity $k$ and an element $c$ of $\mathcal{C}$, we define the size of $c$ in a switching $S$ as the number of contraction nodes of logical complexity $k$ connected to $c$ through their conclusion in $S$. The size of $c$ is the sum over all the switchings $S$ containing $c$ of the sizes of $c$ in $S$. The size of $\mathcal{C}$ is the sum of the sizes of its elements. An important point is that two elements of $\mathcal{C}$ never appear in the same $S$. We show that reducing a cut in $\mathcal{C}$ generates a $\mathcal{C}^{\prime}$ of strictly smaller size and some additional cut links with strictly smaller logical complexity.

\section{Sequentialization}

Sequentialization is proved by showing that an expansion procedure allows us to unfold any of our proof nets into a (unique) Girard proof net.

\subsection{Expansion}

DEFINITION 7 Let $\langle\pi, E\rangle$ be a proof structure and p a variable, then:

- the dependency graph of $p$ w.r.t. $\pi$, denoted by $\mathcal{G}_{\pi}^{p}$, is the sub-graph of $\pi$ depending on $p$ (we take only those nodes and edges whose weights contain $p$ or $\bar{p}$ ): its pending edges are called auxiliary doors except the premises of the $\&_{p}$ nodes (if any) which are called main doors;

- the spreading of a weight $v$ over a sub-graph $\mathcal{G}$ of $\pi$, denoted by $v \cdot[\mathcal{G}]$, is the graph $\mathcal{G}$ in which we replace the weight $w$ of any node of $\mathcal{G}$ with the (product) weight vw;

- the restriction $\left\langle\pi_{p}, E\right\rangle$ (resp., $\left.\left\langle\pi_{\bar{p}}, E\right\rangle\right)$ is obtained by replacing in $\pi$ each occurrence of $p$ (resp., $\bar{p})$ with 1 and each occurrence of $\bar{p}$ (resp., $p$ ) with 0 and keeping only those nodes and edges whose weights are still nonzero.

LEMMA 2 (AUXILIARY DOORS) If $\langle\pi, E\rangle$ is a proof structure and $p$ a variable, then all the auxiliary doors of the dependency graph $\mathcal{G}_{\pi}^{p}$ are only premises of contraction nodes.

Proof - It follows from the definition of proof structure; in particular, observe that if the two premises of a $\&_{q}$ node are both doors of a $\mathcal{G}_{\pi}^{p}$, then they must be main doors, i.e, $p=q$ and $\bar{p}=\bar{q}$ (or, resp., $\bar{p}=q$ and $p=\bar{q}$ ).

DEFINITION 8 A proof net $\langle\pi, E\rangle$ is expandable w.r.t. a variable $p$ (also, $p$-expandable) if it contains a node $L$, with

usual: atoms have complexity 1 ; the complexity of $A^{\perp}$ is the same as the complexity of $A$; the complexity of $B \bullet C$, where $\bullet$ is any binary connective, is the sum of the complexities of $B$ and $C$ plus 1 . weight $w$ depending on $p$, for which there does not exist in $\pi$ any node $\&_{p}$, with weight $v$, such that $w \leq v$.

DEFINITION 9 Assume $\langle\pi, E\rangle$ is expandable w.r.t. a variable $p$, then $\left\langle\pi^{\prime}, E\right\rangle$ is the graph obtained by the following expansion step (we write $\langle\pi, E\rangle \stackrel{\exp }{\longmapsto} p\left\langle\pi^{\prime}, E\right\rangle$ for saying that $\langle\pi, E\rangle$ expands to $\left\langle\pi^{\prime}, E\right\rangle$, w.r.t. $\left.p\right)$ :

1. we take the dependency graph $\mathcal{G}_{\pi}^{p}$, with doors $A_{1}, \ldots, A_{n}$;

2. we take the spreading graphs $v_{1} \cdot\left[\mathcal{G}_{\pi}^{p}\right], \ldots, v_{m} \cdot\left[\mathcal{G}_{\pi}^{p}\right]$, where each $v_{i}$, for $1 \leq i \leq m$, is either a weight of a node $\&_{p}$ of $\pi$ or a suffix weight occurring in some equation $\epsilon_{p} @ v_{i}=0$ of $E$;

3. we replace each occurrence of $\overline{\epsilon_{p}}$ by 1 in any nonzero weight of each graph $v_{i} \cdot\left[\mathcal{G}_{\pi}^{p}\right]$, if $v_{i}$ is a suffix weight occurring in some equation $\epsilon_{p} @ v_{i}=0$ of $E$;

4. $\pi^{\prime}$ is obtained by replacing in $\pi$ the graph $\mathcal{G}_{\pi}^{p}$ with the sum of the spreading graphs $v_{1} \cdot\left[\mathcal{G}_{\pi}^{p}\right], \ldots, v_{m} \cdot\left[\mathcal{G}_{\pi}^{p}\right]$ : each auxiliary door $A_{j}$, with $1 \leq j \leq n$, is expanded according to the Figure 17 (the main doors remain untouched).
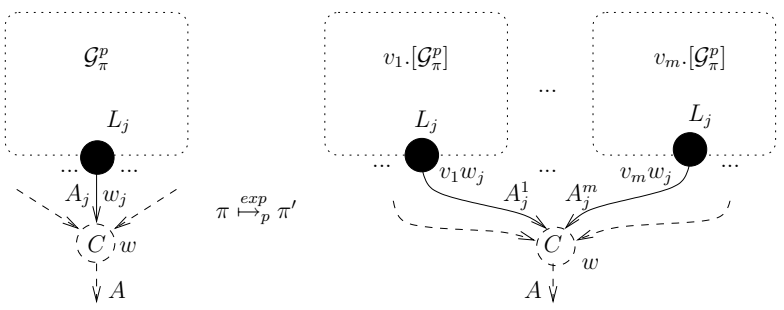

Figure 17. Expansion of an auxiliary door

A proof net that is not expandable (w.r.t. any variable) is said in expanded form.

LEMMA 3 (STABILITY UNDER EXPANSION) If $\langle\pi, E\rangle$ is a proof net that expands in one step to $\left\langle\pi^{\prime}, E\right\rangle$, then $\left\langle\pi^{\prime}, E\right\rangle$ is still a proof net.

Proof - If $\langle\pi, E\rangle \stackrel{\exp }{\longmapsto}\left\langle\pi^{\prime}, E\right\rangle$, then it is easy to verify that $\left\langle\pi^{\prime}, E\right\rangle$ is still a proof structure: in particular observe that, after the $p$-expansion step of the Definition 9 (see Figure 17) in $\left\langle\pi^{\prime}, E\right\rangle$ we have

$$
w_{j}=\left(w_{j} \cdot \sum_{i=1}^{m} v_{i}\right)
$$

since by definition of proof structure, in $\langle\pi, E\rangle$ the weight $w_{j}$ of a door $A_{j}$ depends on $p$ so it must be $w_{j} \leq \sum_{i=1}^{m} v_{i}$, 


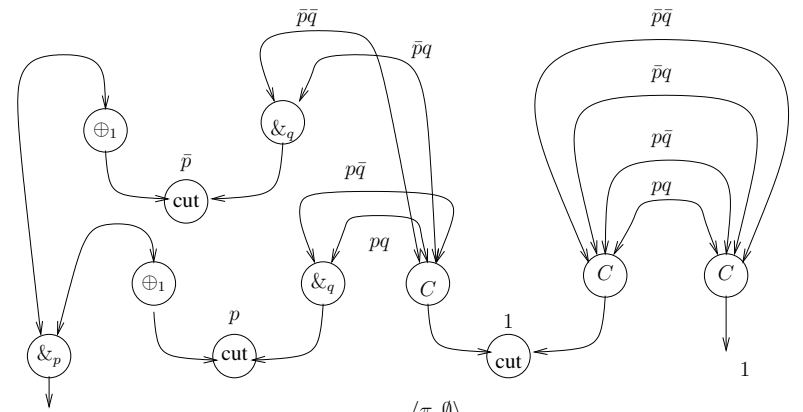

$\langle\pi, \emptyset\rangle$
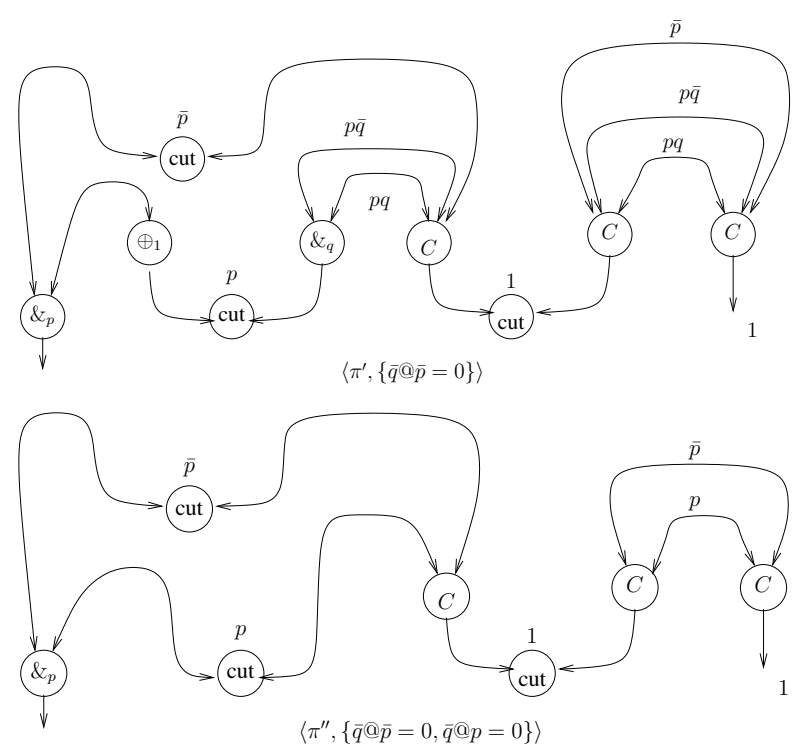

Figure 18. Examples of expansion

modulo $E$; moreover, this sum is a monomial weight, modulo $E$, by definition of PS. Recall that condition 3 of Definition 9 forces us to replace with 0 the occurrence of $\epsilon_{p}$ in the (spreading) product $v_{i} w_{j}$ as soon as $v_{i}$ is the suffix of an equation $\epsilon_{p} @ v_{i}=0$ in $E$; however, this fact has no effect on the above equation 3 , since $v_{i} w_{j}$ is equivalent to $v_{i} w_{j}^{*}$ up to $E$, if $w_{j}^{*}$ is $w_{j}$ after replacing $\epsilon_{p}$ with 0 .

Correctness is easy to prove, since for each switching $S\left(\pi^{\prime}\right)$ we can easily set a switching $S(\pi)$ s.t. $S\left(\pi^{\prime}\right) \subseteq S(\pi)$.

EXAMPLE 3 Observe that the proof structure $\langle\pi, \emptyset\rangle$ of Figure 3 and both the proof structures $\left\langle\pi^{\prime},\{\bar{q} @ \bar{p}=0\}\right\rangle$ and $\left\langle\pi^{\prime \prime},\{\bar{q} @ \bar{p}=0, \bar{q} p=0\}\right\rangle$ of Figure 15 are only expandable w.r.t. q; so, after the q-expansion they transform, respectively, into the proof structure depicted on the top, on the middle and on the bottom of Figure 18.

Note that the notion of dependency graph recalls that one of "empire" defined on Girard's proof nets (see [Gir96]), but dislike this latter, the former one does not constitute a proof structure itself. Moreover, w.r.t. the inclusion relation, if a node $\&_{p}$ of a proof net occurs in the dependency graph of a variable $q$ then in general $\mathcal{G}_{\pi}^{p} \nsubseteq \mathcal{G}_{\pi}^{q}$ : for instance, w.r.t. the proof net of Figure 3, the dependency graph $\mathcal{G}_{\pi}^{p}$ contains the two nodes $\&_{q}$, but $\mathcal{G}_{\pi}^{q} \nsubseteq \mathcal{G}_{\pi}^{p}$; vice-versa, if $\pi$ is in expanded form then, $\mathcal{G}_{\pi}^{q} \subseteq \mathcal{G}_{\pi}^{p}$ (see Example 3).

LEMMA 4 (CONFLUENCE OF THE EXPANSION) Assume $\langle\pi, E\rangle \stackrel{\exp }{\longmapsto}\left\langle\pi_{1}, E\right\rangle$ and $\langle\pi, E\rangle \stackrel{\exp }{\mapsto q}\left\langle\pi_{2}, E\right\rangle$; then there exists a proof net $\left\langle\pi^{*}, E\right\rangle$ s.t. $\left\langle\pi_{1}, E\right\rangle \stackrel{\exp }{\mapsto q}\left\langle\pi^{*}, E\right\rangle$ and $\left\langle\pi_{2}, E\right\rangle \stackrel{\exp }{\longmapsto}\left\langle\pi^{*}, E\right\rangle$.

Proof - Trivially, the expansion is locally confluent.

LEMMA 5 (EXPANDED FORM) If $\langle\pi, E\rangle$ is a proof net in expanded form then it can be mapped into a unique $\mathbf{G i}-$ rard's proof net ${ }^{2} \pi^{*}$.

Proof - Since $\langle\pi, E\rangle$ is a proof net in expanded form, then for each node $L$ with weight $w$ depending on an eigenweight $p$ we know that there exists a unique node $\&_{p}$ node with weight $v$ and s.t. $w \leq v$ (the unicity is due to the fact that the weights of all the possible $\&_{p}$ nodes are pairwise disjoint). This means that the proof structure is almost a Girard's proof structure, if we ignore the possibly unary contraction nodes and the set $E$, that has exhausted its task after the expansion. We need only to make sure that all eigenweights are different. For that, it is enough to iterate the following procedure: we fix an eigenweight $p$ of $\pi$, then if there exists in $\pi$ an unique $\&_{p}$ node we do nothing, otherwise, let $L_{1}$ and $L_{2}$ be two $\&_{p}$ nodes, with weights $w_{1}$, respectively, $w_{2}$; we know that $w_{1} \cdot w_{2}=0$, so there exists at least an occurrence of variable that separates them as follows: let us say $q$, s.t. $q \in w_{1}$ and $\bar{q} \in w_{2}$; then we replace in any weight $w$ of $\pi$ depending on $p$ and s.t. $w \leq w_{1}$, each occurrence of $q p$ (resp., $q \bar{p}$ ) by an occurrence of $q r$ (resp., $q \bar{r}$ ) where $r$ is a new (fresh) eigenweight. We iterate this procedure until all the eigenweights are different in $\pi$. The resulting proof net $\pi^{*}$ is a Girard's proof net. The unicity of this mapping follows by the confluence Lemma 4.

\subsection{Sequentialization}

DEFINITION 10 A proofnet $\langle\pi, E\rangle$, with conclusions $\Gamma$, sequentializes into a proof $\pi^{-}$of $\Gamma$ (we write $\langle\pi, E\rangle \stackrel{\text { seq }}{\longmapsto} \pi^{-}$) if we can inductively map $\langle\pi, E\rangle$ into $\pi^{-}$as follows:

1. $\pi$ is an axiom link with conclusions $A, A^{\perp}$, then, $\pi^{-}$is the axiom $\overline{A, A^{\perp}}$;

2. $\pi$ contains a terminal node $L: \frac{A B}{A \ngtr B}$ s.t. once removed it induces a proof-net $\pi_{1}$ which sequentializes into $\left(\pi_{1}\right)^{-}$with conclusion $\Gamma, A, B$; then $\pi^{-}$is obtained from $\left(\pi_{1}\right)^{-}$by an instance of 8 rule;

\footnotetext{
${ }^{2}$ See the formal Definition 12 in the Appendix A.
} 
3. $\pi$ contains a terminal node $L: \frac{A B}{A \otimes B}$ s.t. once removed it induces two disjoint sequentializable proof-nets (we say, $L$ is splitting): $\pi_{1}$ which sequentializes into $\left(\pi_{1}\right)^{-}$ with conclusion $\Gamma_{1}, A$ and $\pi_{2}$ which sequentializes into $\left(\pi_{2}\right)^{-}$with conclusions $\Gamma_{2}, B$ and $\Gamma=\Gamma_{1} \uplus \Gamma_{2}$; then $\pi^{-}$is obtained from $\pi_{1}^{-}$and $\pi_{2}^{-}$by an instance of $\otimes$ rule;

4. $\pi$ contains a terminal node $L: \frac{A}{A \oplus B}\left(\right.$ resp., $\left.L: \frac{B}{A \oplus B}\right)$ s.t. once removed it induces a proof net $\pi_{1}$ (resp., $\pi_{2}$ ) which sequentializes into $\left(\pi_{1}\right)^{-}$(resp., $\left.\left(\pi_{2}\right)^{-}\right)$with conclusion $\Gamma, A$ (resp., $\Gamma, B)$; then $\pi^{-}$is obtained from $\left(\pi_{1}\right)^{-}$(resp., $\left.\left(\pi_{2}\right)^{-}\right)$by an instance of $\oplus_{1}$ (resp., $\left.\oplus_{2}\right)$ rule;

5. $\pi$ contains a terminal node $L: \frac{A B}{A \&_{p} B}$ s.t. once removed the restrictions $\pi_{p}$ and $\pi_{\bar{p}}$ are still two proof nets (we say, $L$ is splitting) which sequentialize into, respectively, $\left(\pi_{1}\right)^{-}$with conclusions $\Gamma, A$ and $\left(\pi_{2}\right)^{-}$ with conclusions $\Gamma, B$; then $\pi^{-}$is obtained from $\left(\pi_{1}\right)^{-}$ and $\left(\pi_{2}\right)^{-}$by an instance of \& rule.

LEMMA 6 (ADEQUACY) A sequent proof of $\Gamma$ desequentializes into a proof net with same conclusion.

Proof - By induction on the size of the sequent proof.

LEMma 7 (SPLITTING) Let $\langle\pi, E\rangle$ be a proof net that $p$ expands to $\left\langle\pi^{\prime}, E\right\rangle$ and let $L$ be a node that splits $\left\langle\pi^{\prime}, E\right\rangle$ into two sub-proof nets, $\left\langle\pi_{1}^{\prime}, E\right\rangle$ and $\left\langle\pi_{2}^{\prime}, E\right\rangle$; then $L$ also splits $\langle\pi, E\rangle$ into two sub-proof nets $\left\langle\pi_{1}, E\right\rangle$ and $\left\langle\pi_{2}, E\right\rangle$ which p-expand, respectively, to $\left\langle\pi_{1}^{\prime}, E\right\rangle$ and to $\left\langle\pi_{2}^{\prime}, E\right\rangle$.

Proof - Assume $L$ splits $\left\langle\pi^{\prime}, E^{\prime}\right\rangle$ into $\left\langle\pi_{1}^{\prime}, E^{\prime}\right\rangle$ and $\left\langle\pi_{2}^{\prime}, E^{\prime}\right\rangle$; we need to consider two cases, according to $L$.

If $L=\otimes$, then the proof follows by:

FACT 1 There do not exist two $\&_{q}$ nodes, $L_{1}$ with weight $w_{1}$ and $L_{2}$ with weight $w_{2}$, such that $L_{1} \in \pi_{1}^{\prime}$ and $L_{2} \in \pi_{2}^{\prime}$ (or vice-versa).

In order to show this fact, we reason by absurdum, and we assume there exist such two nodes $L_{1}$ and $L_{2}$ in $\pi^{\prime}$; moreover, we assume $\pi^{\prime}$ is minimal w.r.t. the number of possible expansion steps; then there must exist a variable $r$ that separates $w_{1}$ and $w_{2}$, i.e., $r \in w_{1}$ and $\bar{r} \in w_{2}$ (or vice-versa), since, by definition of proof structure, $w_{1} \cdot w_{2}=0$. Now, w.r.t. $r,\left\langle\pi^{\prime}, E^{\prime}\right\rangle$ is either expandable or not. The former case contradicts the assumption of minimality (actually, it is not difficult to verify that if $L$ is splitting in $\pi$ then it will be still splitting in $\pi^{\prime}$ after an expansion step). In the second case there must exist a node $\&_{r}$ with weight $w$ such that $w_{1} \leq w$ and $w_{2} \leq w$; since $\pi^{\prime}$ is splitting, this node $\&_{r}$ must occur either in $\pi_{1}^{\prime}$ or in $\pi_{2}^{\prime}$ : the case $\&_{r}$ occurs in $\pi_{1}^{\prime}$ implies that either $\pi_{2}^{\prime}$ is not a proof structure, in case $r$ is not prefix of any equation in $E$, or $\pi_{2}^{\prime}$ is expandable w.r.t. $r$, contradicting the assumption of minimality.

Fact 1 implies that $L$ also splits $\pi$ into $\pi_{1}$ and $\pi_{2}$ which, trivially, $p$-expand to $\pi_{1}^{\prime}$, respectively, $\pi_{2}^{\prime}$.

If $L=\&_{r}$, then the proof follows by:

FACT 2 There do not exist two $\&_{q}$ nodes, $L_{1}$ with weight $w_{1}$ and $L_{2}$ with weight $w_{2}$, such that $L_{1} \in \pi_{1}^{\prime}$ and $L_{2} \in \pi_{2}^{\prime}$ (or vice-versa) except when $r$ occurs in $w_{1}$ and $\bar{r}$ in $w_{2}$ (or vice-versa) and $w_{i=1,2} \leq w(L)=1$.

In order to show this fact we reason like in the previous case: we assume by absurdum such two nodes $L_{1}$ and $L_{2}$; then there exists a variable $s$ that separates $w_{1}$ and $w_{2}$. Now, either $\left\langle\pi^{\prime}, E^{\prime}\right\rangle$ is expandable w.r.t. $s$ or not. The former case contradicts the assumption of minimality. In the second case there exists a node $\&_{s}$ whose weight $w$ is such that $w_{1} \leq w$ and $w_{2} \leq w$; if $r=s$, we have done; otherwise, since $\pi^{\prime}$ is splitting, \&s must occur either in $\pi_{1}^{\prime}$ or in $\pi_{2}^{\prime}$ : the case $\&_{s}$ occurs in $\pi_{1}^{\prime}$ implies that either $\pi_{2}^{\prime}$ is not a proof structure, in case $r$ is not prefix of any equation in $E^{\prime}$, otherwise $\pi_{2}^{\prime}$ must be expandable w.r.t. $s$, contradicting the assumption of minimality. Moreover, observe that if $p=r$ then the terminal node $\&_{p}$ must be unique, since it weight is 1 by definition.

Fact 2 implies that $L$ also splits $\pi$ into $\pi_{1}$ and $\pi_{2}$ which, trivially, $p$-expand to $\pi_{1}^{\prime}$, respectively, $\pi_{2}^{\prime}$.

\section{LEMMA 8 (SEQUENTIALIZATION UNDER EXPANSION)} Let $\langle\pi, E\rangle$ be a proof net that expands in one step to a proof net $\left\langle\pi^{\prime}, E\right\rangle$ that sequentializes into $\left(\pi^{\prime}\right)^{-}$; then $\langle\pi, E\rangle$ also sequentializes into $\left(\pi^{\prime}\right)^{-}$.

Proof - Assume $\pi$ expands w.r.t. $p$ to $\pi^{\prime}$ and $\pi^{\prime}$ sequentializes into $\left(\pi^{\prime}\right)^{-}$, then we proceed by induction on the size (the number of nodes) of $\pi$ (we omit the set $E$ ). The case when $\pi$ is an axiom is trivial. Otherwise, by assumption, we know there exists a terminal node $L$ that once removed from $\pi^{\prime}$ it induces (at least) one sub-proof net $\pi_{i}^{\prime}$ (with $1 \leq i \leq 2$ ) which sequentializes into $\left(\pi_{i}^{\prime}\right)^{-}$. If we show, case by case according to $L$, that any graph $\pi_{i}$ obtained by removing the conclusion node $L$ from $\pi$ is also a proof net that expands to $\pi_{i}^{\prime}$, then we can apply the induction hypothesis and conclude that $\pi$ also sequentializes into $\left(\pi^{\prime}\right)^{-}$following the schema below:

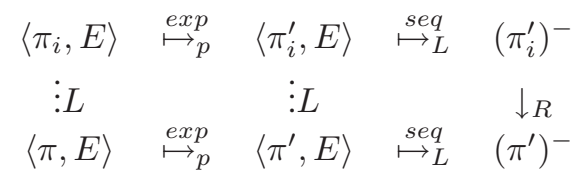

If $L$ is a 8 , or an $\oplus_{i}$ node then it cannot be an expanded node, otherwise since $L$ is a terminal node it should be either a node $\&_{p}$ or a $C$ node, contradicting our assumption. The cases when $L$ is a 8 or a $\oplus_{i}$ node are easy and we omit them. 
Otherwise, $L$ is a splitting $\otimes$ or $\&_{q}$ node, then by Definition 10 we know that $\pi^{\prime}$ splits into two sub-proof nets $\pi_{1}^{\prime}$ and $\pi_{2}^{\prime}$, therefore, by the Splitting Lemma 7, we know that $\pi$ also splits into two proof nets $\pi_{1}$ and $\pi_{2}$ which expand, respectively, to $\pi_{1}^{\prime}$ and to $\pi_{2}^{\prime}$; then, by induction hypothesis we know that $\pi_{1}$ and $\pi_{2}$ sequentialize, respectively, into $\left(\pi_{1}^{\prime}\right)^{-}$ and into $\left(\pi_{2}^{\prime}\right)^{-}$; therefore, $\pi$ sequentializes into $\left(\pi^{\prime}\right)^{-}$.

THEOREM 4 (SEQUEnTIALIZATION) If $\langle\pi, E\rangle$ is a proof net with conclusion $\Gamma$, then it sequentializes into a sequent proof $\pi^{-}$with same conclusions.

Proof - We iteratively expand $\langle\pi, E\rangle$ until we get, by Lemma 3, a proof net $\left\langle\pi^{\prime}, E^{\prime}\right\rangle$ in expanded form which can be mapped, by Lemma 5, to a Girard proof net $\pi^{*}$ with the same conclusions $\Gamma$; now, by Girard's sequentialization (see [Gir96]) we know that $\pi^{*}$ sequentializes into a sequent proof $\left(\pi^{*}\right)^{-}$of $\Gamma$, so by Lemma 8 we conclude that also $\langle\pi, E\rangle$ sequentializes into $\left(\pi^{*}\right)^{-}$.

\section{Conclusions}

The main contribution of this paper is the first definition of a full cut elimination procedure for monomial proof nets for multiplicative additive linear logic based on a new possibility of sharing nodes by reusing eigenweights.

As opposed to the definition of proof nets by Hughesvan Glabbeek, our extension of Girard's definition does not immediately lead to a canonical representation of proofs. However the kind of sharing we propose should be compatible with the introduction of the exponential connectives and may allow us to revisit the theory of proof nets for full linear logic.

Another use of sharing is given by the Geometry of Interaction $(\mathrm{GoI})$. We have to evaluate the impact on GoI of our new sharing capabilities.

Finally the complexity of the extended correctness criterion and of the cut elimination procedure have to be studied.

\section{References}

[Gir87] Girard, J-Y. Linear Logic. Theoretical Computer Science, 50, pp.1-102, 1987.

[Gir96] Girard, J-Y. Proof-nets: the parallel syntax for proof theory. Logic and Algebra. Marcel Dekker, 1996.

[HvG03] Hughes, D. and Van Glabbeek, R. Proof Nets for Unit-free Multiplicative-Additive Linear Logic. In Proc. of IEEE Logic in Computer Science, 2003.
[Lau99] Laurent, O. Polarised Proof-Nets: Proof-Nets for LC (Extended Abstract). In J.-Y. Girard, editor, Typed Lambda Calculi and Applications 1999, LNCS 1581, pp. 213-227. SpringerVerlag. Avril 1999.

\section{A Appendix: Girard's proof structures}

DEFINITION 11 A proof structure à la Girard (GPS) is a pre-proof structure with weights associated as follows:

1. first we associate a Boolean variable, called eigenweight $p$, to each \&-node (eigenweights are supposed to be different);

2. then we associate a weight, a product of (negation of) Boolean variables $(p, \bar{p}, q, \bar{q} \ldots)$ to each node, with the constraint that two nodes have the same weight if they have a common edge, except when the edge is the premise of a \& or C-node, in these cases we do like in Figure 2:

3. a conclusion node has weight 1 ;

4. if $w$ is the weight of a \&-node, with eigenweight $p$, and $w^{\prime}$ is a weight depending on $p$ and appearing in the proof structure then $w^{\prime} \leq w$ (a weight $w$ depends on $p$ when $p$ or $\bar{p}$ occurs in $w)$.

DEFINITION 12 A GPS $\pi$ is correct, so it is a proof net à la Girard (GPN), if any switching, induced by any valuation of $\pi$, is acyclic and connected (ACC).

THEOREM 5 A GPN is sequentialized into a MALL sequent proof with same conclusions and vice-versa. 